\title{
Influence of macro-scale environmental variables on diversity and distribution pattern of lichens in Badrinath valley, Western Himalaya.
}

\section{Gupta $S^{1,2}$, Khare $R^{1,3}$, Rai $H^{1,3}$, Upreti DK ${ }^{3}$, Gupta RK ${ }^{1}$, Sharma PK $^{2}$, Srivastava $\mathrm{K}^{4}$ and Bhattacharya $\mathrm{P}^{5}$}

\author{
${ }^{1}$ Department of Botany Pt. L.M.S. Government Post Graduate College, Rishikesh (Dehradun), Uttarakhand-249201, \\ India. \\ ${ }^{2}$ Department of Environmental Science, Graphic Era University, 566/6, Bell Road, Clement Town, Dehradun, \\ Uttarakhand-248002, India. \\ ${ }^{3}$ Lichenology laboratory; Plant Diversity, Systematics and Herbarium Division; CSIR-National Botanical research \\ Institute, Lucknow, Uttar Pradesh-226001, India. \\ ${ }^{4}$ Information System and Services Division, Indian Meteorological Department, Ministry of Earth Sciences, Mausam \\ Bhavan, Lodhi Road, New Delhi- 110003, India. \\ ${ }^{5}$ University School of Environment Management, GGS Indraprastha University, Block-A, Sector- 16 C, Dwarka, New \\ Delhi-110078, India.
}

Gupta S, Khare R, Rai H, Upreti DK, Gupta RK, Sharma PK, Srivastava K, Bhattacharya P 2014 Influence of macro-scale environmental variables on diversity and distribution pattern of lichens in Badrinath valley, Western Himalaya. Mycosphere 5(1), 229-243, Doi 10.5943/mycosphere/5/1/12

\begin{abstract}
Morphological growth forms confer ecological adaptability to lichens species and are indicators of habitat conditions and various climatic as well as zooanthropogenic pressures. Lichens samples from six sites in two locations of Badrinath valley were studied in order to assess the influence of macro-scale environmental variables (i.e. altitude, relative humidity and temperature) on diversity and distribution of lichens, using ordination (PCA and hierarchical clustering) and correlation analysis. The study recorded 106 lichen species in the valley. Parmeliaceae was a dominant family. Lichen species constitution in sites resulted in different groups, which were determined by the dominant growth forms and substrate preferences. Lichen growth form distribution was significantly correlated with studied macro-scale environment variables. On rock (saxicolous) substrate was the main substrate of lichen inhabitancy in the valley. The study concluded that macro-scale environmental variables play determining role in lichen community constitution of alpine habitats in Himalayas.
\end{abstract}

Key words - Cluster analysis - crustose - dimorphic - foliose - fruticose -Parmaliaceae principal component analysis - saxicolous - terricolous

\section{Introduction}

Lichens, mutualistic associations of a dominant fungus (mycobiont) and a green (phycobiont) and/or blue-green algae (cyanobiont), are by far known as one of the most successful symbionts in nature (Galloway 1992). Lichen thallus is a relatively stable and well-balanced symbiotic system with both heterotrophic and autotrophic components and is often regarded as a self-contained miniature ecosystem (Farrar 1976, Seaward 1988). 
Lichens exhibit a wide range of morphological growth forms which confer lichen with ecological efficiency to adapt according to various climatic as well as zooanthropogenic stresses (Ahti 1959, Sheard 1968, Ahti et al. 1973, Rai et al. 2012a, b). The dominance of specific lichen growth forms in a habitat is outcome of multiple interacting environmental factors which ultimately decide the constitution of a lichen community (Eldridge and Rosentreter 1999, Zedda et al. 2011, Rai et al 2012 a, b). Himalayan habitats are regions with harsh climate, characterized by regular orographic precipitation, longer periods of snow fall, higher UV radiation and freezing minimum temperatures (Rai et al. 2012 a, b, Khare et al. 2010). Lichen growth forms in such alpine habitats in Himalayas are indictors of habitat variability, and change in macro- scale environmental variables i.e. altitude, relative humidity and temperature (Rai et al. $2012 \mathrm{a}, \mathrm{b}$ )

The present study deals with the influence of macroscale environmental variables (altitude, temperature and relative humidity) on diversity and distribution pattern of lichens in an alpine habitat of Garhwal Himalayas.

\section{Materials \& Methods}

\section{Study area}

Badrinath valley is situated in north-west extreme (N30 44'1.43" E79 29'37.4"N30 46'33.74" E79 ${ }^{0} 29^{\prime} 32.08^{\prime \prime}$ ) of Chamoli district in the state of Uttarakhand, India (Fig. 1). Situated in the floodplains of Alaknanda river (a tributary of Ganges), the valley houses the temple of Badrinath, one of the most important pilgrimage sites among the four Hindu Charm Dhams. With an average elevation of $3,300 \mathrm{~m}$, the valley is situated in outer Himalaya and represents typical alpine habitats; characterized by alpine-grassland vegetation, extreme temperature and precipitation regimes.

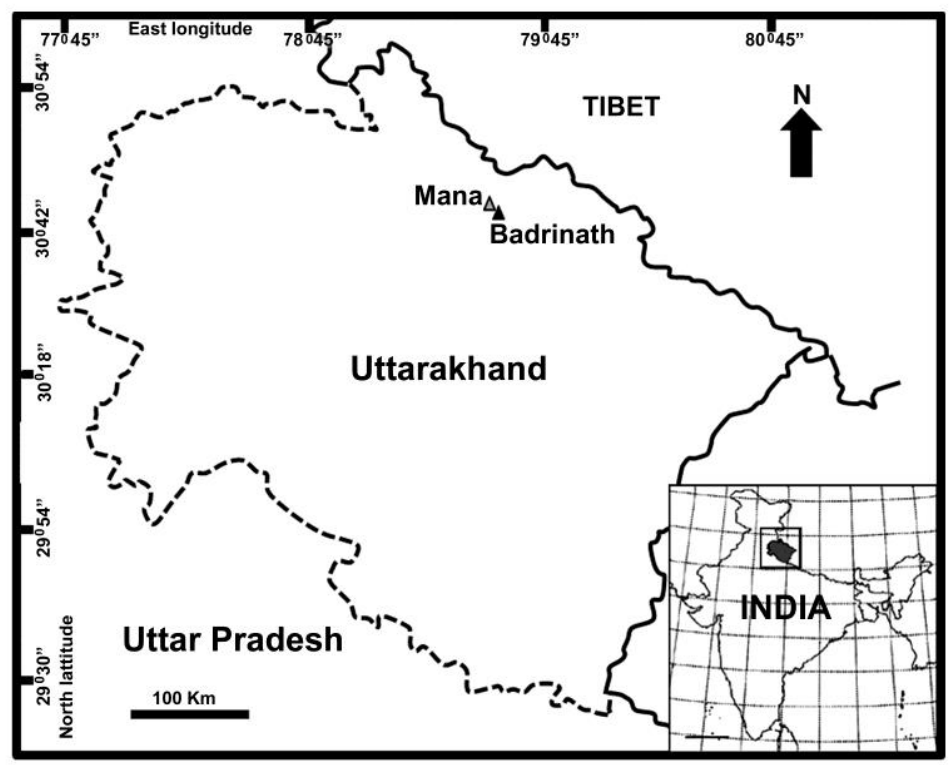

Fig. 1 - Location map of study locales in Badrinath valley.

The landscape of the area is typically mountainous, with steep slopes (46-60 ) and elevation rising from 2,950 to 3,670 m. The topography comprises ridges and exposed rocks with patches of inclined alpine grassland. Precipitation occurs as snow, hail, heavy rain and showers. Snowfall occurs from December to March, and snowmelt occurs during April and May- providing an abundance of soil water prior to the monsoon period. Maximum rainfall is in July-Oct (Indian Meteorological department- IMD) (Fig. 2). 


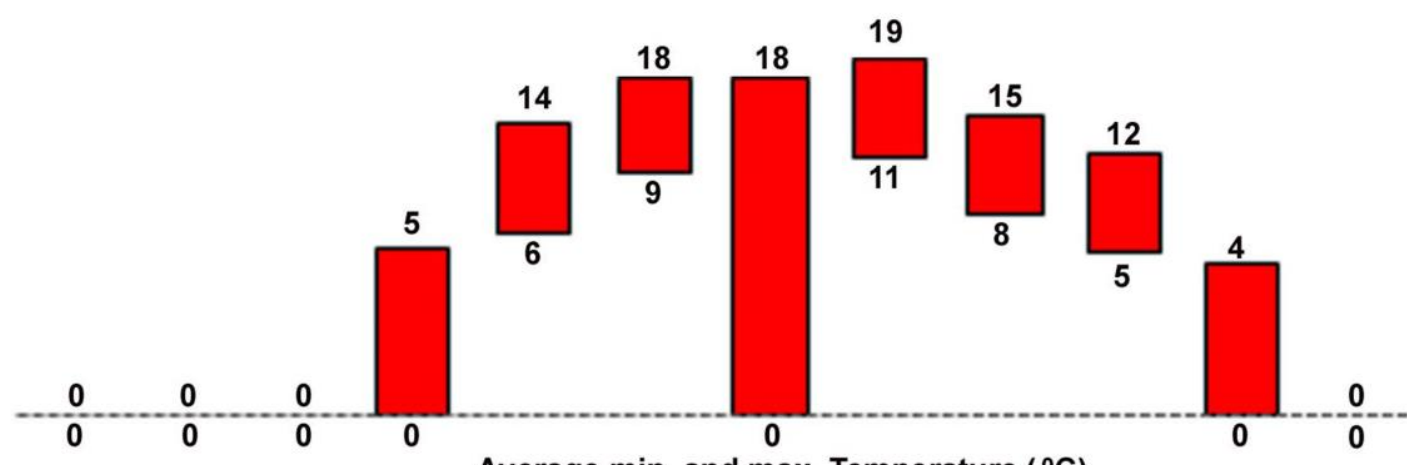

Average min. and max. Temperature $\left({ }^{\circ} \mathrm{C}\right)$

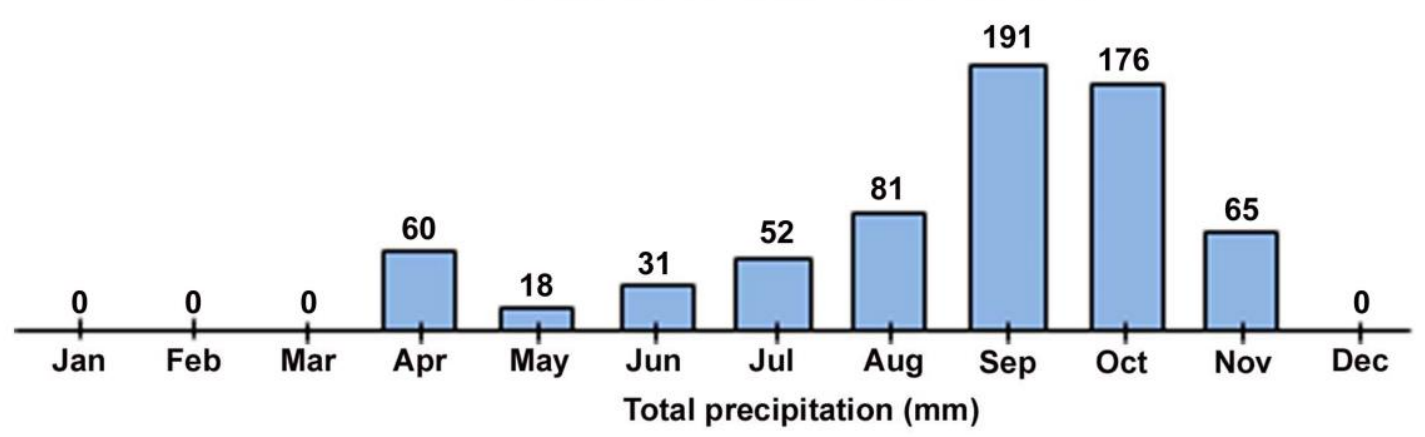

Fig. 2 - Climograph of Badrinath valley showing average annual temperature regime and annual total precipitation

The maximum monthly temperatures in the area vary from $18-19^{\circ} \mathrm{C}$ in the months of JuneAug, while minimum temperatures as low as $-22^{\circ} \mathrm{C}$ are recorded during December-February (Fig. 2). The vascular plant vegetation is broadly alpine, with alpine scrub or grassland dominated by crataegus (Hawthorns) species with sporadic presence of tree species of Betula, Salix and stunted Rhododendron.

Landuse by the local human population is mainly semipastoral agriculture based on livestock grazing, agriculture, and the collection of fodder and fuel from alpine grasslands. Open grasslands (Bugyals) at 2,900-3,400 m are extensively used as pasturelands by native populations and nomads (Bhotiyas). In addition, land around the Badrinath shrine and at Mana is occupied during the pilgrimage season. The most favoured microhabitats for lichens in the area are rocks, soil patches amongst rocks, soil in rock crevices and woody shrubs.

The study was conducted on lichen samples of two locations in Badrinath valley- in and around (i) Badrinath town (N30 44'35.41" E79 $\left.29^{\circ} 9^{\prime} 38.18^{\prime \prime}\right)$ and (ii) Mana town (N30 46’21.55" E $79^{\circ} 29^{\prime} 43.86$ ") (Fig. 1), deposited in lichenology herbarium (LWG) of CSIR-National Botanical Research Institute (CSIR-NBRI), Lucknow, Uttar Pradesh, India.

\section{Data recording}

The study recognized three major sites each, in both of the localities (Table 1). Data of all macroscale environmental variables (altitude, temperature and relative humidity) were recorded either from herbarium records (altitude) or from climate data obtained from IMD. Vegetation of sampling sites was recorded during recent collections (2012-13).

Unidentified and freshly collected lichen samples were examined and identified upto species level using a stereomicroscope, light microscope (morpho-anatomically), and chemically with the help of spot tests, UV light and standardized thin-layer chromatography (Elix and ErnstRussel 1993, Orange et al. 2001). Keys and monographs employed for authentication included those of Ahti (2000), Awasthi (2007), Saag et al. (2009) and Rai et al. (2014). Data regarding lichen species diversity at all 6 collection sites of the two localities and their growth form diversity was carefully recorded. 
Table 1 Macroscale environmental variables and vegetation attributes of sampling sites of the study area.

\begin{tabular}{|c|c|c|c|c|c|c|}
\hline \multirow[t]{2}{*}{ Locations } & \multirow[t]{2}{*}{$\begin{array}{l}\text { Average } \\
\text { Altitude* } \\
\text { (m) }\end{array}$} & \multirow[t]{2}{*}{ Sites $^{\dagger}$} & \multicolumn{2}{|c|}{$\begin{array}{l}\text { Average } \\
\text { Temperature* } \\
\left({ }^{0} \mathrm{C}\right) \\
\end{array}$} & \multirow{2}{*}{$\begin{array}{l}\text { Average } \\
\text { Relative } \\
\text { humidity* } \\
(\%)\end{array}$} & \multirow[t]{2}{*}{ Vegetation } \\
\hline & & & Min. & Max. & & \\
\hline Badrinath & $3180 \pm 0.5$ & $\begin{array}{l}\text { Badrinath town } \\
\text { (B1) } \\
\text { at Devdarshini(B2) } \\
\text { Brahmini } \\
\text { Village(B3) }\end{array}$ & $12 \pm 1.1$ & $24 \pm 0.2$ & $66 \pm 0.5$ & $\begin{array}{l}\text { Mainly dominated by shrub species } \\
\text { of Berberis, Cotoneaster, Rosa, and } \\
\text { Juniperus; herbs species of } \\
\text { Gentiana, Swertia, Anaphalis, } \\
\text { Morina, Tanacetum, Gaultheria, } \\
\text { Nepeta, Cyananthus, Potentilla, } \\
\text { Thalictrum, Rumex, Thymus, } \\
\text { Dracocephalum, Astragalus, } \\
\text { Pedicularis, Saussurea along with } \\
\text { stunted Rhododendron and tree } \\
\text { species of Hipphophae and Betula } \\
\text {. }\end{array}$ \\
\hline Mana & $3420 \pm 1.1$ & $\begin{array}{l}\text { Mana (M1) } \\
\text { Bhimpul (M2) } \\
\text { enroute Vasudhara } \\
\text { falls(M3) }\end{array}$ & $8 \pm 0.5$ & $18 \pm 0.2$ & $55 \pm 0.3$ & $\begin{array}{l}\text { Mainly dominant by shrub species } \\
\text { of Berberis alongwith herbaceous } \\
\text { species of Anaphalis, Gentiana, } \\
\text { Swertia, Nepeta, Bistorta, Sedum, } \\
\text { Rosularia, Thalictrum, Oxyria, } \\
\text { Rhodila, Taraxacum, and sporadic } \\
\text { presence of few trees of Betula and } \\
\text { Salix. }\end{array}$ \\
\hline
\end{tabular}

*Values are in mean \pm standard deviation; ${ }^{\dagger}$ abbreviations for each site are reported in parentheses.

\section{Data analysis}

An indirect gradient ordination method, principal component analysis (PCA), was used to summarize the compositional differences between the sites (Gauch 1982, ter Braak and Prentice 1988, Rai et al. 2012). Two tailed bivariate correlation analysis was performed by calculating Pearson's correlation coefficients to compare explanatory variables- i.e. altitude, temperature, relative humidity) and response variables- i.e. growth forms (Pinokiyo et al. 2008, Rai et al. 2012 b). Lichen groups from both Badrinath and Mana were sought through hierarchical cluster analysis (Ludwig and Reynolds 1988, Jongman et al. 1995, Rai et al. 2011, 2012b) using presence (1) absence (0) data matrices, employing Raup-Crick similarity measure (Rai et al. 2011) and unweighted pair-group moving average (UPGMA) algorithm, on two criteria: lichen growth form diversity and their substratum of occurrence (i.e. on rock, on soil, on soil over rock, on twig and on cement plaster) (Scutari et al. 2004, Rai et al. 2012a, b). The PCA and cluster analysis were performed using multivar option in PAST 2.17c (Hammer et al. 2001, Rai et al 2012b); all the other above mentioned statistical analyses were done using IBM SPSS Statistics ver. 20.

\section{Results}

The study recorded occurrence of 106 lichen species from the 6 sites of two localities. All the six sites of the two localities support a diverse, but somewhat different assemblage of lichen species both in terms of species and growth form constitution.

Macroscale variables (i.e. minimum, maximum temperature and percentage relative humidity) of the landscape varied along the altitudinal gradient; there was a gradual decrease observed in the ambient temperature (maximum and minimum) and relative humidity (\%) from Badrinath town to Mana (Table 1).

\section{Taxonomic diversity}

The lichen assemblage at Badrinath consisted of 57 species belonging to 29 genera and 19 families. Peltigeraceae (9 spp.) and Parmeliaceae (8 spp.) were dominant families followed by 
Physciaceae, and Teloschistaceae (Table 2) at Badrinath. The lichen assemblage at Mana consisted of 58 species belonging to 33 genera and 18 families. Parmeliaceae (11spp.) and Physciaceae (9 spp.) were dominant families followed by Acarosporaceae, and Teloschistaceae (Table 2) at Mana. Among both the locations, Badrinath exhibits dominance of cyanolichen genera (Peltigera, Leptogium, Stereocaulon, Nephroma) than Mana (Nephroma, Stereocaulon).

\section{Growth form distribution patterns}

Four growth forms i.e. crustose (squamulose and leprose are treated as subtypes; Nash III 2002), foliose, fruticose and dimorphic (squamules as primary thallus bearing erect fruticose body as secondary thallus) were recorded at both locations (i.e. Badrinath and Mana). Among the different growth forms foliose dominated, followed by crustose, fruticose and dimorphic growth forms. While the relative proportion of crustose growth form was higher in Mana than Badrinath, the other two growth forms i.e. fruticose and dimorphic were higher in proportion in Badrinath than Mana (Table 2; Fig 4, 5).

\section{Lichen community composition (PCA analysis)}

The PCA analysis required 5 components (axis) to account for $100 \%$ variation in the data set. The first two axes explained 78\% (axis 1, 60 and axis 2, 18\% respectively) of variance in the study (Fig. 3). Among the 6 sites of the two localities where, sites B2, B3, M2 and M1 formed cluster showing somewhat similar species constitution, sites M3 and B1 were with totally different species constitution (Fig. 3). Sites B3 and M2 clustered due to the presence of nearly equal proportion of both foliose and crustose growth forms whereas sites B2 and M1 fall apart due to greater proportion of crustose growth forms in B2 and foliose in M1(Table 2). Sites M3 and B1 clustered apart significantly due to distinct dominance of crustose and foliose growth forms respectively in these sites (Table 2, Fig. 3).

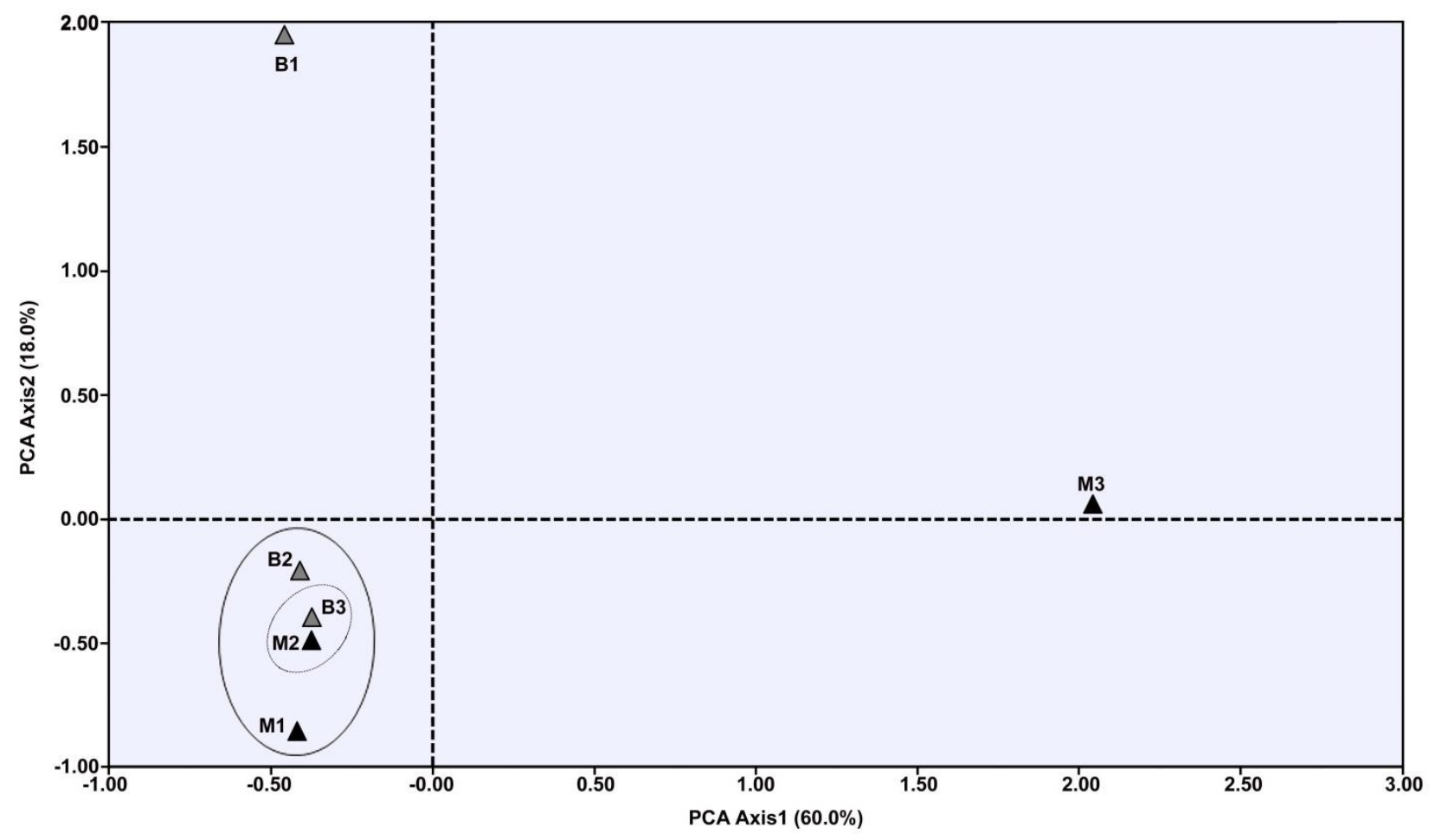

Fig. 3 - PCA ordination plot of 6 study sites in two locations of Badrinath valley-Badrinath and Mana towns, (refer Table 1 for abbreviations). 
Table 2 Lichen species recorded in the Six sampling in Badrinath and Mana town location in Badrinath valley

\begin{tabular}{|c|c|c|c|c|c|c|c|c|c|}
\hline \multirow[t]{2}{*}{ Species } & \multirow[t]{2}{*}{ Family } & \multicolumn{3}{|c|}{ Badrinath } & \multicolumn{2}{|c|}{ Mana } & \multirow{2}{*}{\multicolumn{2}{|c|}{ Substratum }} & \multirow{2}{*}{$\begin{array}{l}\text { Gro } \\
\text { wth } \\
\text { For } \\
\mathbf{m}^{\dagger}\end{array}$} \\
\hline & & $\begin{array}{l}\mathbf{B} \\
\mathbf{1}\end{array}$ & $\begin{array}{l}\text { B } \\
2\end{array}$ & $\begin{array}{l}\text { B } \\
\mathbf{3}\end{array}$ & $\begin{array}{l}M \\
1\end{array}$ & $\begin{array}{l}\mathbf{M} \\
\mathbf{2}\end{array}$ & & & \\
\hline Acarospora angolensis $\mathrm{H}$. Magn. & Acarosporaceae & - & - & - & + & - & - & On rock & $\mathrm{Cr}$ \\
\hline Acarospora bullata Anzi. & Acarosporaceae & - & - & - & + & - & - & On rock & $\mathrm{Sq}^{*}$ \\
\hline Acarospora carnegiei Zahlbr. & Acarosporaceae & - & - & - & - & + & - & On rock & $\mathrm{Cr}$ \\
\hline Acarospora oxytona (Ach.)Massal. & Acarosporaceae & - & - & - & - & - & + & On rock & $\mathrm{Cr}$ \\
\hline Acarospora saxicola Fink. & Acarosporaceae & - & - & - & + & - & - & On rock & $\mathrm{Cr}$ \\
\hline Acarospora scabra (Pres.) T. Fries & Acarosporaceae & - & - & - & + & - & - & On rock & $\mathrm{Sq}^{*}$ \\
\hline $\begin{array}{l}\text { Acarospora smaragdula (wahlenb } \\
\text { in Ach.) Massal. }\end{array}$ & Acarosporaceae & + & - & - & - & - & + & On rock & $\mathrm{Cr}$ \\
\hline Aspicilia griseocinerea Räsänen & Megasporaceae & - & + & - & - & - & - & On soil over rock & $\mathrm{Cr}$ \\
\hline $\begin{array}{l}\text { Aspicilia almorensis (Räsänen). } \\
\text { Awasthi }\end{array}$ & Megasporaceae & + & - & - & - & - & - & On rock & $\mathrm{Cr}$ \\
\hline $\begin{array}{l}\text { Aspicilia alphoplaca (Wahlenb.in. } \\
\text { Ach) Poelt }\end{array}$ & Megasporaceae & - & - & - & + & - & - & On rock & $\mathrm{Cr}$ \\
\hline Aspicilia calcarea (L.). Mudd & Megasporaceae & - & - & - & + & + & - & On rock & $\mathrm{Cr}$ \\
\hline $\begin{array}{l}\text { Aspicilia maculata (Magn.) D.D. } \\
\text { Awasthi }\end{array}$ & Megasporaceae & - & - & - & - & + & - & On rock & $\mathrm{Cr}$ \\
\hline Buellia aethalea (Ach.) Th. Fr. & Caliciaceae & + & - & - & - & - & - & On rock & $\mathrm{Cr}$ \\
\hline Buellia asterella Poelt \& M. Sulzer & Caliciaceae & - & - & - & - & - & + & On soil & $\mathrm{Cr}$ \\
\hline $\begin{array}{l}\text { Caloplaca flavovirescens (Wulfen) } \\
\text { Dalla. Torre \& Sarnth. }\end{array}$ & Teloschistaceae & - & - & - & + & - & + & On rock & $\mathrm{Cr}$ \\
\hline Caloplaca lithophila Mangn. & Teloschistaceae & - & - & - & + & - & - & On rock & $\mathrm{Cr}$ \\
\hline Caloplaca subsoluta (Nyl.) Zahlbr. & Teloschistaceae & + & - & - & - & - & - & On rock & $\mathrm{Cr}$ \\
\hline $\begin{array}{l}\text { Canomaculina } \\
\text { subtinctoria (Zahlbr.) Elix }\end{array}$ & Parmeliaceae & + & - & - & - & - & - & On rock & Fo \\
\hline $\begin{array}{l}\text { Candelaria concolor (Dicks.) } \\
\text { Arnold }\end{array}$ & Candelariaceae & + & - & - & - & - & - & On rock & $\mathrm{Cr}$ \\
\hline Candelaria indica (Hue) Vain & Candelariaceae & - & - & - & - & + & - & On rock & Fo \\
\hline $\begin{array}{l}\text { Candelariella aurella (Hoffm.) } \\
\text { Zahlber. }\end{array}$ & Candelariaceae & + & - & - & - & - & - & On rock & $\mathrm{Cr}$ \\
\hline $\begin{array}{l}\text { Candelariella grimmiae Poelt \& } \\
\text { Reddi }\end{array}$ & Candelariaceae & - & - & - & - & - & + & Cement Plaster & $\mathrm{Cr}$ \\
\hline Cladonia borealis S.Stenroos & Cladoniaceae & + & - & - & - & - & - & On soil over rock & $\mathrm{Dm}$ \\
\hline Cladonia cartilaginea Krempelh. & Cladoniaceae & - & + & - & - & - & - & On soil over rock & $\mathrm{Dm}$ \\
\hline Cladonia furcata (Huds.)Schards & Cladoniaceae & - & - & - & - & - & + & On soil & $\mathrm{Dm}$ \\
\hline Cladonia mongolica Ahti. & Cladoniaceae & - & + & - & - & - & - & On rock & $\mathrm{Dm}$ \\
\hline Cladonia pyxidata (L.) Hoffm. & Cladoniaceae & - & - & - & - & - & + & On soil & $\mathrm{Dm}$ \\
\hline $\begin{array}{l}\text { Dermatocarpon miniatum (L.) W. } \\
\text { Mann }\end{array}$ & Verrucariaceae & + & + & + & - & - & - & On soil over rock & Fo \\
\hline $\begin{array}{l}\text { Dermatocarpon vellereum } \\
\text { Zschacke. }\end{array}$ & Verrucariaceae & - & - & - & + & + & - & On rock & Fo \\
\hline Dimelaena oreina (Ach.) Norman & Caliciaceae & - & - & - & + & + & - & On rock & $\mathrm{Cr}$ \\
\hline $\begin{array}{l}\text { Diploschistes gypsaceus (Arch.) } \\
\text { Nyl. }\end{array}$ & Thelotremataceae & - & - & - & - & + & - & On rock & $\mathrm{Cr}$ \\
\hline $\begin{array}{l}\text { Diploschistes muscorum (Scop.)R. } \\
\text { Sant. }\end{array}$ & Thelotremataceae & - & - & + & - & - & + & On soil over rock & $\mathrm{Cr}$ \\
\hline $\begin{array}{l}\text { Diploschistes rampoddensis } \\
\text { (Nyl.)Zahlbr. }\end{array}$ & Thelotremataceae & + & - & - & - & - & - & On rock & $\mathrm{Cr}$ \\
\hline $\begin{array}{l}\text { Diploschistes scruposus (Schreb.) } \\
\text { Norman }\end{array}$ & Thelotremataceae & + & - & - & - & + & - & On rock & $\mathrm{Cr}$ \\
\hline
\end{tabular}




\begin{tabular}{|c|c|c|c|c|c|c|c|c|c|}
\hline \multirow[t]{2}{*}{ Species } & \multirow[t]{2}{*}{ Family } & \multicolumn{3}{|c|}{ Badrinath } & \multicolumn{2}{|c|}{ Mana } & \multirow{2}{*}{\multicolumn{2}{|c|}{$\begin{array}{ll} & \text { Substratum } \\
\text { M } & \\
\mathbf{3} & \end{array}$}} & \multirow{2}{*}{$\begin{array}{l}\text { Gro } \\
\text { wth } \\
\text { For } \\
\text { m }^{\dagger}\end{array}$} \\
\hline & & $\begin{array}{l}\mathbf{B} \\
\mathbf{1}\end{array}$ & $\begin{array}{l}\text { B } \\
2\end{array}$ & $\begin{array}{l}\text { B } \\
\mathbf{3}\end{array}$ & $\begin{array}{l}\mathbf{M} \\
\mathbf{1}\end{array}$ & $\begin{array}{l}\mathbf{M} \\
2\end{array}$ & & & \\
\hline $\begin{array}{l}\text { Endocarpon subrosettum Ajay } \\
\text { Singh \& Upreti }\end{array}$ & Verrucariaceae & - & - & - & + & - & - & On rock & $\mathrm{Cr}$ \\
\hline Evernia mesomorpha Nyl. & Parmeliaceae & - & - & - & - & - & + & On twigs & $\mathrm{Fr}$ \\
\hline Flavoparmelia caperata (L.) Hale & Parmeliaceae & + & - & - & - & + & - & On rock & Fo \\
\hline $\begin{array}{l}\text { Flavopunctelia flavertior (stirton) } \\
\text { Hale }\end{array}$ & Parmeliaceae & - & - & - & - & - & + & On twigs & Fo \\
\hline $\begin{array}{l}\text { Heterodermia diademata (Toylor). } \\
\text { Awasthi }\end{array}$ & Physciaceae & - & - & - & + & - & - & On fallen twigs & Fo \\
\hline $\begin{array}{l}\text { Heterodermia hypocaesia (Yasudu) } \\
\text { Awasthi }\end{array}$ & Physciaceae & + & - & - & - & - & - & On soil over rock & Fo \\
\hline $\begin{array}{l}\text { Heterodermia leucomelos (L.) } \\
\text { Poelt. }\end{array}$ & Physciaceae & - & - & - & - & - & + & On soil over rock & Fo \\
\hline $\begin{array}{l}\text { Heterodermia pseudospeciosa } \\
\text { (Wulfen) Trevis. }\end{array}$ & Physciaceae & + & - & - & - & - & + & On soil over rock & Fo \\
\hline Lasallia pertusa (Rass.) Llano & Umbilicariaceae & + & - & - & - & - & + & On rock & Fo \\
\hline Lasallia pustulata (L.) Mérat & Umbilicariaceae & + & - & - & - & - & - & On rock & Fo \\
\hline $\begin{array}{l}\text { Lecanora muralis (Schreb.) } \\
\text { Rabenh. }\end{array}$ & Lecanoraceae & + & + & + & + & - & - & On soil over rock & $\mathrm{Cr}$ \\
\hline Lecanora phadrophthalma Poelt & Lecanoraceae & - & + & - & - & - & - & On soil over rock & $\mathrm{Cr}$ \\
\hline Lecanora pseudistera Nyl. & Lecanoraceae & + & - & - & - & - & - & On rock & $\mathrm{Cr}$ \\
\hline Lecidea confluens (Weber) Ach. & Lecideaceae & - & - & + & - & - & - & On soil over rock & $\mathrm{Cr}$ \\
\hline Lecidea lapicida (Ach.) Ach. & Lecideaceae & - & - & + & - & - & - & On soil over rock & $\mathrm{Cr}$ \\
\hline Lepraria lobificans Nyl. & Stereocaulaceae & - & - & - & + & - & - & On rock & $\mathrm{Lp}^{*}$ \\
\hline Leptogium burnetiae C.W. Dodge & Collemataceae & + & - & - & - & - & - & On rock over soil & Fo \\
\hline $\begin{array}{l}\text { Lobothalia praeradiosa (Nyl.)Poelt } \\
\text { \& Leuck. }\end{array}$ & Megasporaceae & + & + & + & + & - & + & On soil over rock & Fo \\
\hline $\begin{array}{l}\text { Lobothallia alphoplaca (Wahlenb.) } \\
\text { Hafellner }\end{array}$ & Megasporaceae & + & - & - & - & - & - & On soil over rock & Fo \\
\hline Melanelia panniformis (Nyl.) Essl. & Parmeliaceae & - & - & - & - & - & + & On soil & Fo \\
\hline Melanelia villosella ( Ess1.) Essl. & Parmeliaceae & - & - & - & - & - & + & On soil over rock & Fo \\
\hline Nephroma parile (Ach.) Ach. & Nephromataceae & - & - & + & - & - & - & On soil over rock & Fo \\
\hline Nephroma helveticum Ach. & Nephromataceae & - & - & - & - & - & + & On soil over rock & Fo \\
\hline Peltigera canina $(\mathrm{L}$.$) Willd$ & Peltigeraceae & + & - & - & - & - & - & $\begin{array}{l}\text { On soil over rock/ } \\
\text { On soil }\end{array}$ & Fo \\
\hline $\begin{array}{l}\text { Peltigera didactyla (With.) J.R. } \\
\text { Laundon }\end{array}$ & Peltigeraceae & + & - & - & - & - & - & On soil & Fo \\
\hline Peltigera elisabethae Gyeln. & Peltigeraceae & + & - & - & - & - & - & On soil over rock & Fo \\
\hline Peltigera lepidophora (Nyl.) Bitter & Peltigeraceae & + & - & - & - & - & - & On soil & Fo \\
\hline Peltigera malacea (Ach.) Funck & Peltigeraceae & + & - & - & - & - & - & On soil over rock & Fo \\
\hline Peltigera membranacea (Ach.) Nyl. & Peltigeraceae & + & - & - & - & - & - & On soil & Fo \\
\hline $\begin{array}{l}\text { Peltigera praetextata (Flörke ex } \\
\text { Sommerf.) Zopf }\end{array}$ & Peltigeraceae & + & - & - & - & - & - & On soil & Fo \\
\hline Peltigera rufescens (Weiss) Humb. & Peltigeraceae & + & - & - & - & - & - & On soil & Fo \\
\hline Pertusaria leucosora Nyl. & Pertusariaceae & - & - & - & + & - & - & On rock & Fo \\
\hline $\begin{array}{l}\text { Phaeophyscia ciliata (Hoffm.) } \\
\text { Moberg }\end{array}$ & Physciaceae & - & - & - & + & - & - & On rock & Fo \\
\hline Phaeophyscia hispidula (Ach.)Essl. & Physciaceae & - & - & - & + & + & + & On rock $\backslash$ on twigs & Fo \\
\hline $\begin{array}{l}\text { Phaeophyscia orbicularis (Necker) } \\
\text { Moberg }\end{array}$ & Physciaceae & - & - & - & + & + & - & On rock & Fo \\
\hline $\begin{array}{l}\text { Phaeophyscia primaria (Poelt) } \\
\text { Trass }\end{array}$ & Physciaceae & - & - & - & + & - & - & On soil over rock & Fo \\
\hline Physcia biziana (A. Massal.) & Physciaceae & + & - & - & - & - & - & On soil over rock & Fo \\
\hline
\end{tabular}




\begin{tabular}{|c|c|c|c|c|c|c|c|c|c|}
\hline \multirow[t]{2}{*}{ Species } & \multirow[t]{2}{*}{ Family } & \multicolumn{3}{|c|}{ Badrinath } & \multicolumn{2}{|c|}{ Mana } & \multirow[b]{2}{*}{$\begin{array}{l}\mathbf{M} \\
\mathbf{3}\end{array}$} & \multirow[t]{2}{*}{ Substratum } & \multirow{2}{*}{$\begin{array}{l}\text { Gro } \\
\text { wth } \\
\text { For } \\
\mathbf{m}^{\dagger} \\
\end{array}$} \\
\hline & & $\begin{array}{l}\text { B } \\
1\end{array}$ & $\begin{array}{l}\text { B } \\
2\end{array}$ & $\begin{array}{l}\mathbf{B} \\
\mathbf{3}\end{array}$ & $\begin{array}{l}\mathbf{M} \\
\mathbf{1}\end{array}$ & $\begin{array}{l}\mathbf{M} \\
2\end{array}$ & & & \\
\hline $\begin{array}{l}\text { Physcia caesia (Hoffm.) Hampe ex } \\
\text { Fürnr. }\end{array}$ & Physciaceae & + & + & - & - & - & - & On soil over rock & Fo \\
\hline Physcia leptalea (ach.) DC & Physciaceae & - & - & + & - & - & - & On soil over rock & Fo \\
\hline Physconia detersa (Nyl.) Poelt. & Physciaceae & - & - & - & - & - & + & On rock & Fo \\
\hline $\begin{array}{l}\text { Physconia enteroxantha (Nyl.) } \\
\text { Poelt. }\end{array}$ & Physciaceae & - & - & - & - & + & - & On soil over rock & Fo \\
\hline Physconia muscigena (Ach.) Poelt & Physciaceae & + & - & - & - & - & - & On soil over rock & Fo \\
\hline $\begin{array}{l}\text { Porpidia crustulata (Ach.) Hertel \& } \\
\text { Knoph }\end{array}$ & Porpidiaceae & - & - & + & - & - & - & On soil over rock & $\mathrm{Cr}$ \\
\hline Punctelia borreri $(\mathrm{Sm}.) \mathrm{Krog}$ & Parmeliaceae & - & - & - & + & - & - & On rock & Fo \\
\hline $\begin{array}{l}\text { Punctelia rudecta (Taylor) Elix \& J. } \\
\text { Johnst. }\end{array}$ & Parmeliaceae & + & - & - & - & - & - & On rock & Fo \\
\hline $\begin{array}{l}\text { Rhizocarpon disporum (Naeg.ex. } \\
\text { Hepp)Müll. Arg }\end{array}$ & Rhizocarpaceae & - & - & - & - & + & - & On rock & $\mathrm{Cr}$ \\
\hline Rhizocarpon geographicum (L.) DC & Rhizocarpaceae & + & - & - & + & - & + & On rock & $\mathrm{Cr}$ \\
\hline Rhizocarpon macrosporum Räsänen & Rhizocarpaceae & - & - & + & + & - & - & On rock & $\mathrm{Cr}$ \\
\hline Rhizocarpon sublucidum Räsänen & Rhizocarpaceae & - & + & + & - & - & - & On rock & $\mathrm{Cr}$ \\
\hline Rhizoplaca chrysoleuca (Sm.) Zopf. & Lecanoraceae & + & - & + & - & + & + & On rock & $\mathrm{Cr}$ \\
\hline Stereocaulon foliolosum $\mathrm{Nyl}$. & Stereocaulaceae & - & - & - & - & - & + & On rock & $\mathrm{Fr}$ \\
\hline Stereocaulon alpinum Laurer & Stereocaulaceae & + & - & - & - & - & - & On soil over rock & Fr \\
\hline Stereocaulon myriocarpum Th.Fr. & Stereocaulaceae & - & + & + & - & - & - & On soil over rock & $\mathrm{Fr}$ \\
\hline $\begin{array}{l}\text { Tephromela khatiensis ( Räsänen) } \\
\text { Lumbsch }\end{array}$ & Tephromelataceae & + & - & - & + & - & - & On rock & $\mathrm{Cr}$ \\
\hline Umbilicaria indica var. indica Frey & Umbilicariaceae & + & - & - & - & - & - & On rock & Fo \\
\hline Umbilicaria vellea (L.) Ach. & Umbilicariaceae & + & - & - & - & - & - & On rock & Fo \\
\hline Verrucaria acrotella Ach. & Verrucariaceae & - & - & - & + & - & - & On rock & $\mathrm{Cr}$ \\
\hline $\begin{array}{l}\text { Xanthoparmelia bellatula } \\
\text { (Kurok.\& Filson) Elix \& al. }\end{array}$ & Parmeliaceae & - & - & - & + & - & - & On soil over rock & Fo \\
\hline $\begin{array}{l}\text { Xanthoparmelia australasica D.J. } \\
\text { Galloway }\end{array}$ & Parmeliaceae & - & - & - & + & - & - & On rock & Fo \\
\hline $\begin{array}{l}\text { Xanthoparmelia coreana (Gyeln.) } \\
\text { Hale }\end{array}$ & Parmeliaceae & + & - & - & - & - & - & On rock & Fo \\
\hline $\begin{array}{l}\text { Xanthoparmelia mexicana (Gyeln.) } \\
\text { Hale. }\end{array}$ & Parmeliaceae & + & - & - & - & - & - & On soil over rock & Fo \\
\hline $\begin{array}{l}\text { Xanthoparmelia somloensis } \\
\text { (Gyeln.)Hale. }\end{array}$ & Parmeliaceae & + & - & - & - & - & - & On soil over rock & Fo \\
\hline $\begin{array}{l}\text { Xanthoparmelia stenophylla (Ach.) } \\
\text { Ahti \& Hawksw. }\end{array}$ & Parmeliaceae & + & - & - & - & - & + & On soil over rock & Fo \\
\hline $\begin{array}{l}\text { Xanthoparmelia taractica (Kremp.) } \\
\text { Hale }\end{array}$ & Parmeliaceae & + & - & - & - & - & - & On soil over rock & Fo \\
\hline $\begin{array}{l}\text { Xanthoparmelia terricola Hale. } \\
\text { Nash \& Elix in Hale }\end{array}$ & Parmeliaceae & - & - & - & - & - & + & On rock & Fo \\
\hline $\begin{array}{l}\text { Xanthoparmelia tinctina (Maheu \& } \\
\text { A. Gillet) Hale }\end{array}$ & Parmeliaceae & - & - & - & + & - & - & On rock & Fo \\
\hline Xanthoria candelaria (L.) Th.Fr. & Teloschistaceae & + & + & - & - & - & - & On soil over rock & Fo \\
\hline Xanthoria elegans (Link.)Th.Fr. & Teloschistaceae & + & - & - & + & + & + & On rock & Fo \\
\hline $\begin{array}{l}\text { Xanthoria fallax var. subsorediosa } \\
\text { (Räsänen) D.D. Awasthi }\end{array}$ & Teloschistaceae & + & - & - & - & - & - & On soil over rock & Fo \\
\hline Xanthoria sorediata (Vainio). Poelt & Teloschistaceae & - & - & + & + & + & + & On soil over rock & Fo \\
\hline Xanthoria ulophyllodes Räsänen & Teloschistaceae & + & - & - & + & - & - & On rock $\backslash$ on twigs & Fo \\
\hline
\end{tabular}




\section{Correlations}

Among the macroscale variables analyzed altitude, relative humidity, minimum and maximum temperature were significantly correlated with diversity of the four growth forms recorded, indicating a gradual increase in crustose growth forms than others (e.g. foliose, fruticose, and dimorphic) with increasing altitude; decreasing relative humidity and ambient atmospheric temperature (Table 3). The diversity of foliose, fruticose, and dimorphic growth forms was negatively correlated with crustose (Table 3). Relative humidity and minimum and maximum temperatures negatively correlated to the altitude (Table 3 ).

\section{Lichen groups}

Hierarchical cluster analysis resulted in three major clusters (I-III) in Badrinath and two in Mana(I, II) (Fig. 4, 5). In both locations the clustering clearly demarcated lichen communities based on growth forms, which were further differentiated on the basis of substratum preferences. In Badrinath the major cluster I consisted of fruticose and dimorphic species (Stereocaulon and Cladonia), whereas the major clusters II and III consisted of foliose and crustose growth forms respectively (Fig. 4). In Mana the major cluster I consisted mainly of foliose growth forms, whereas the major clusters II consisted of crustose growth form (Fig. 5). Cladonia pyxidata appears as an outlier in Mana as it was the only species with dimorphic growth form. These major clusters in both locations were further subclustered according to their substrate preferences. In both locations saxicolous (on rock) substratum dominated over the all other substratums (Fig. 4, 5). Diversity of crustose lichen was higher in Mana than Badrinath (Fig. 4, 5).

Table 3 Pearson's correlation coefficients for selected variables (significant correlations are tagged).

\begin{tabular}{llllllll}
\hline & Cr & Fo & Fr & Dm & Rh & Alt & MinT \\
\hline Fo & $-1.000^{*}$ & & & & & & \\
Fr & $-1.000^{*}$ & $1.000^{*}$ & & & & \\
Dm & $-1.000^{*}$ & $1.000^{*}$ & $1.000^{*}$ & & & \\
Rh & $-1.000^{*}$ & $1.000^{*}$ & $1.000^{*}$ & $1.000^{*}$ & & & \\
Alt & $1.000^{*}$ & $-1.000^{*}$ & $-1.000^{*}$ & $-1.000^{*}$ & $-1.000^{*}$ & & \\
MinT & $-1.000^{*}$ & $1.000^{*}$ & $1.000^{*}$ & $1.000^{*}$ & $1.000^{*}$ & $-1.000^{*}$ & \\
MaxT & $-1.000^{*}$ & $1.000^{*}$ & $1.000^{*}$ & $1.000^{*}$ & $1.000^{*}$ & $-1.000^{*}$ & $1.000^{*}$ \\
\hline
\end{tabular}

*Correlation is significant at the 0.01 level (2-tailed).

Variables: $\mathrm{Cr}=$ crustose growth form, $\mathrm{Fo}=$ foliose growth form, $\mathrm{Fr}=$ fruticose growth form, $\mathrm{Dm}=$ dimorphic growth form, $\mathrm{Rh}=$ percentage average relative humidity, Alt $=$ altitude, $\mathrm{MinT}=$ average annual minimum temperature, MaxT= average minimum temperature.

\section{Discussion}

Organism's occurrence in a habitat is the function of limited range of various environmental variables, and within these range they are found to be most abundant, indicating their specific environmental optimum (Körner 2003). Altitude linked macro-scale environmental variables such as relative humidity, temperature and precipitation influence both the taxonomic as well as growth form diversity of lichen communities, worldwide (Ahti 1964, John and Dale 1990, Wolf 1993, Pirintsos et al 1995, Upreti and Negi 1998, Grytnes et al 2006, Pinokiyo et al 2008). The phytogeographical distribution of Indian lichens is found to be influenced by climatic factors which are derived from elevation gradients throughout the country (Upreti 1998). Himalayan habitats due to higher environment lapse rate coupled with increasing zooanthropogenic pressures constitute some of the most fragile ecosystems. Lichen communities in Himalaya are characterized by specific growth forms and habitat subsets along elevational gradients (Upreti 1998). Lichens in 


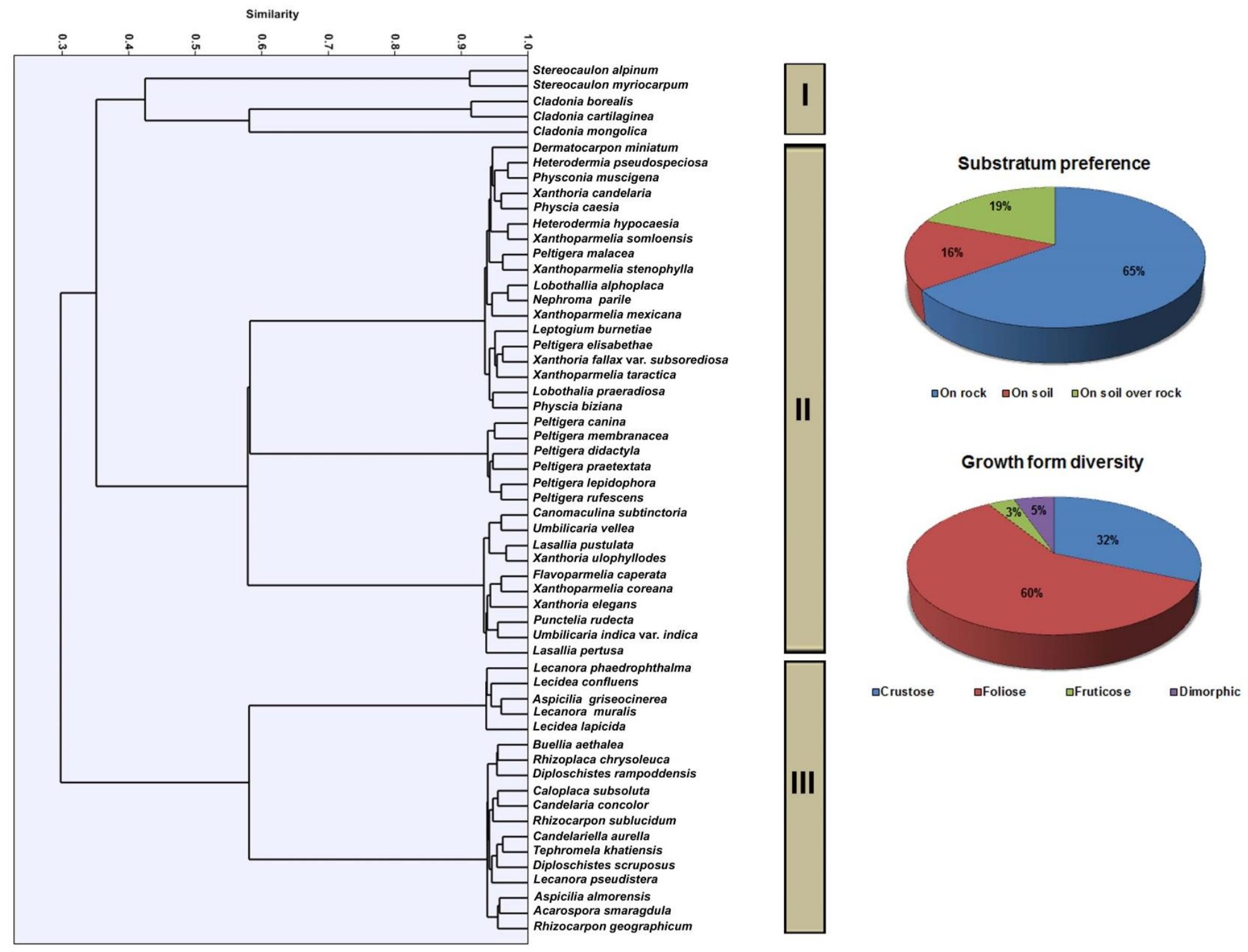

Fig. 4 - Groups of lichen species in and around Badrinath town resulting from hierarchical cluster analysis based on growth form diversity and substrate preferences. 

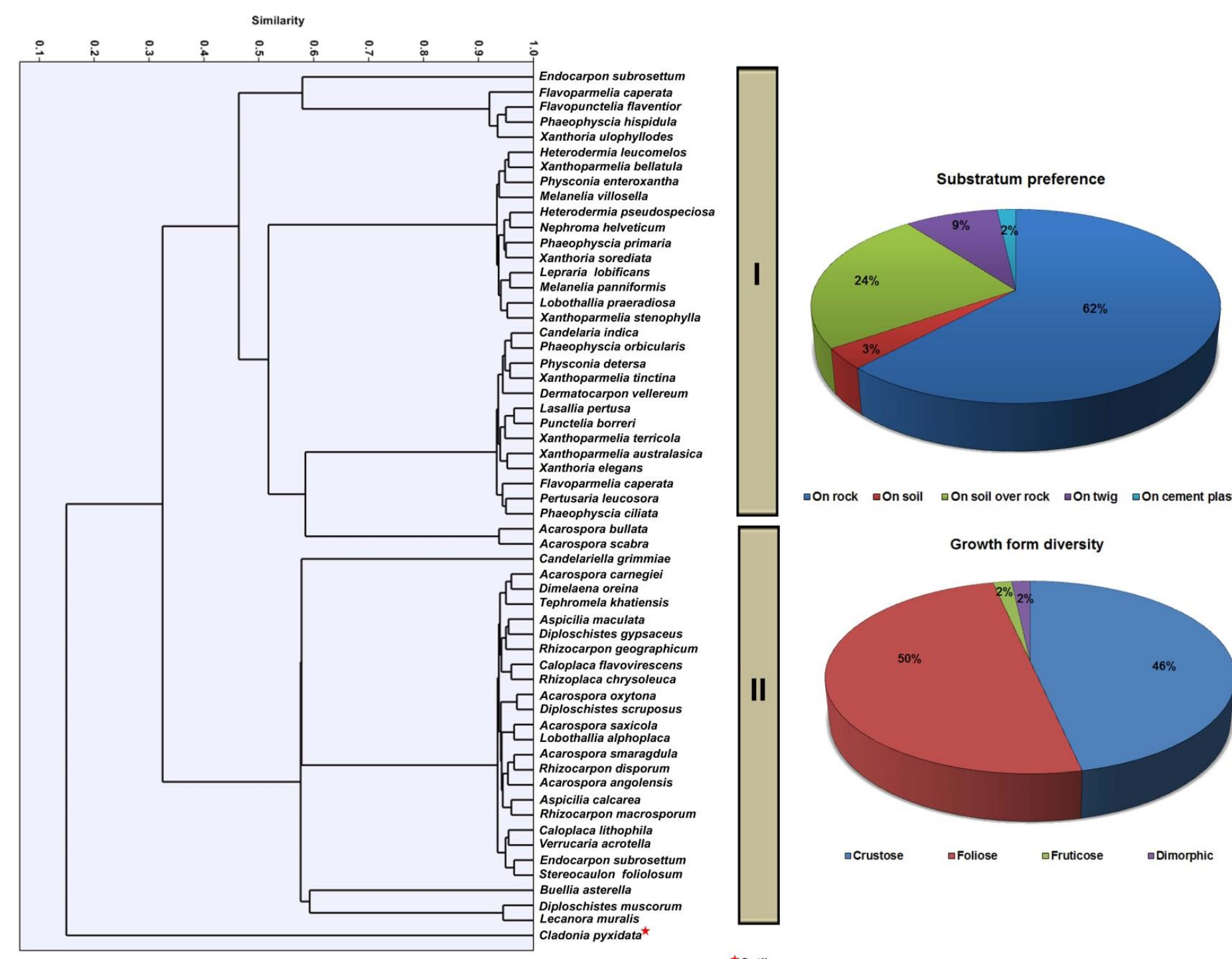

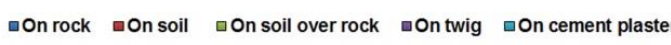
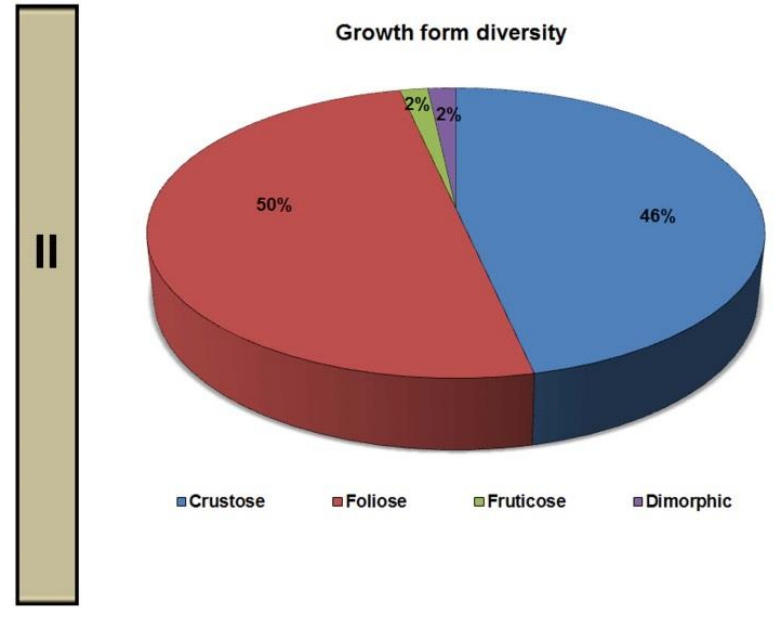

toutier

Fig. 5 - Groups of lichen species in and around Mana town resulting from hierarchical cluster analysis based on growth form diversity and substrate preferences. 
Himalayas are dominated by epiphytic, foliose growth forms upto $3000 \mathrm{~m}$, followed by terricolous and saxicolous; foliose, fruticose and crustose growth forms at higher altitudes $(\geq 3200 \mathrm{~m})$ (Negi 2000, Negi and Gadgil 2002, Negi and Upreti 2000, Upreti and Negi 1998). Lichen communities above treeline $(\geq 3500 \mathrm{~m}$ ) in Himalayas are influenced by both availability of suitable substrate and severity of harsh climates, which allow specific tolerant growth forms to survive (Negi 2000, Negi and Upreti 2000, Rai et al 2012 a, b, Rai 2012).

Badrinath valley, situated in alpine regions of Himalayas, by virtue of its stressed climatology (i.e. higher environmental lapse rate, high wind velocity, high UV radiation, low atmospheric pressure and low precipitation), and delimiting nutrient and exposure regime, support relatively simple ecosystems, characterized by limited trophic levels and relatively very few plant growth forms and species (Rai et al 2010). Absence of dominant growth of angiospermic vascular plants in the valley reduces vegetative competition, which allows lichens to flourish and inhabit all available relevés. The overall dominance of Parmeliaceae over other families in both locations (i.e Badrinath and Mana) is a characteristic feature of Himalayan lichens (Upreti 1998, Negi 2000, Negi and Upreti 2000). The richness of cyanolichen species in Badrinath than Mana can be attributed to higher moisture and less harsh temperature regime in Badrinath (Blum 1973, Kershaw 1985, Lange et al 1986, Lange 2003, Rai et al. 2013). Though foliose growth form dominated in Badrinath valley, other characteristic terricolous (soil inhabiting) growth forms of alpine Himalayan habitats, i.e. fruticose (Stereocaulon) and dimorphic (Cladonia) (Rosentreter et al. 2014) were delimited to Badrinath town, due to greater soil cover which tends to decrease towards Mana (Rai et al 2012b). As shown by correlation analysis the comparative increase in proportion of crustose growth forms in Mana can be attributed to increasing altitude, low relative humidity and lower atmospheric temperature in mountainous habitats of Mana (Dietrich and Scheidegger 1996, 1997, Shrestha and St. Clair 2011). The comparative clustering of the six sampling sites of the two localities in PCA reflects the constitutional difference in lichen growth forms in these sites, which are influenced by environmental as well as land scape (soil cover) variables (Lalley 2006, Rai et al 2012b). The hierarchical clustering of lichen species at both the locations in Badrinath valley concluded that lichen communities in the valley form natural clusters based on growth forms which are further differentiated in two sub-clusters according to substrate variability (Scutari et al. 2004, Rai et al 2012b). As the harsh climate of the valley is able to sustain small diversity of other ground flora, rock and soil over rock was the dominant substrate for lichens in the valley (Rai et al. 2012b). The clustering exemplifies the role of altitude linked changes in macro-scale environmental variable which influence the comparative abundance of different lichen growth forms (Rai et al 2012b).

It is evident from the above observations that lichen communities in alpine habitats of Himalaya are influenced both by macro-scale environmental as well as land scape variables, which cluster lichens according to ecologically viable growth form groups over period of time. Broad similar land use in both the locations of study in Badrinath valley, suggest that the environmental variables which sustain the growth of lichens species must be monitor and conserved as lichen communities play crucial role in stabilizing the ecology of the region.

\section{Acknowledgements:}

Authors are grateful to Director, CSIR-National Botanical Research Institute, Lucknow for providing necessary laboratory facilities. The study of Himanshu Rai was supported by Uttarakhand State Council for Science and Technology, through MRD project grant (UCOSTUCS\&T/R\&D/LS-26/11-12/4370 dated 17-03-2012).

\section{References}

Ahti T. 1959 - Studies on the caribou lichen stands of Newfoundland. Annales Botanici Societatis Zoologicae Botanicae Fennicae Vanamo 30, 1-43.

Ahti T. 1964 - Macrolichens and their zonal distribution in boreal and arctic Ontario, Canada. 
Annales Botanici Fennici 1, 1-35.

Ahti T. 2000 - Cladoniaceae. [Flora neotropica monograph 78]. New York Botanical Garden Press, New York.

Ahti T, Scotter GW, Vänskä H. 1973 - Lichens of the Reindeer Preserve, Northwest Territories, Canada. The Bryologist 76, 48-76.

Awasthi DD. 2007 - A compendium of the macrolichens from India, Nepal and Sri Lanka. Bishen Singh Mahendra Pal Singh, Dehra Dun.

Blum OB. 1973 - Water relations. In: Ahmadjian V, Hale ME, (eds). The lichens. New York: Academic Press, p.381-400.

Dietrich M, Scheidegger C. 1996 - The importance of sorediate crustose lichens in the epiphytic lichen flora of the swiss plateau and the Pre-Alps. Lichenologist 28, 245-256.

Dietrich M, Scheidegger C. 1997 - Frequency, diversity and ecological strategies of epiphytic lichens in the Swiss Central Plateau and the Pre-Alps. Lichenologist 29, 237-258.

Eldridge D, Rosentreter R 1999 - Morphological groups: a framework for monitoring microphytic crusts in arid landscapes. Journal of Arid Environments 41, 11-25.

Elix JE, Ernst-Russel KD. 1993 - A catalogue of standardized thin layer chromatographic data and biosynthetic relationships for lichen substances, 2nd edn. Australian National University, Canberra.

Farrar JF. 1976 - The lichen as an ecosystem: observation and experiment. In: D. H. Brown, D. L. Hawksworth and R. H. Bailey (eds), Lichenology: Progress and Problems, pp. 385-406. Academic Press, London.

Galloway DJ. 1992 - Biodiversity: a lichenological perspective. Biodiversity and Conservation 1,, 312-323.

Gauch HG JR. 1982 - Multivariate analysis in community structure. Cambridge University Press, Cambridge.

Grytnes JA, Heegaard E, Ihlen PG. 2006 - Species richness of vascular plants, bryophytes, and lichens along an altitudinal gradient in western Norway. Acta Oecologica 29, 241-246.

Hammer $\varnothing$, Harper DAT, Ryan DP. 2001 - PAST: Paleontological statistics software package for education and data analysis. Palaentologia Electronica 4, 1-9. (http://palaeoelectronica.org/2001_1/past/past.pdf)

John E, Dale MRT. 1990 - Environmental correlates of species distributions in a saxicolous lichen community. Journal of Vegetation Science 1, 385-392.

Jongman RHG, ter Braak CJF, van Tongeren OFR (eds) 1995- Data analysis in community and landscape ecology. Cambridge University Press, Cambridge.

Kershaw KA. 1985 - The lichen environment: moisture. In: Kershaw KA, Physiological ecology of lichens, pp. 30-53, Cambridge University Press, Cambridge.

Khare R, Rai H, Upreti DK, Gupta RK. 2010 - Soil Lichens as indicator of trampling in high altitude grassland of Garhwal, Western Himalaya, India. Fourth National Conference on Plants \& Environmental Pollution, 8-11 Dec.2010, p.135-136.

Körner C. 2003 - Alpine Plant Life - Functional Plant Ecology of High Mountain Ecosystems, 2. ed., Springer, Heidelberg, p.344.

Lalley JS, Viles HA, Copeman N, Cowley C 2006 - The influence of multi-scale environmental variables on the distribution of terricolous lichens in a fog desert. Journal of Vegetation Science 17, 831-838.

Lange OL. 2003 - Photosynthesis of soil-crust biota as dependent on environmental factors. In: Belnap J, Lange OL (Eds) Biological soil crusts: structure, function, and management [Ecological Studies, vol. 150]. Springer-Verlag, Berlin, Heidelberg, pp 217-240.

Lange OL, Kilian E, Ziegler H. 1986 - Water vapor uptake and photosynthesis of lichens: performance differences in species with green and blue-green algae as photobionts. Oecologia 71, 104-110.

Ludwig JA, Reynolds JF. 1988 - Statistical ecology. A primer on methods and computing. John Wiley, London. 
Nash III TH, Ryan BD, Gries C, Bungartz F. 2002 - Lichen flora of the Greater Sonoran Desert region, vol 1. Lichens unlimited, Arizona State University, Tempe, AZ. p. 532.

Negi HR 2000 - On the patterns of abundance and diversity of macrolichens of Chopta-Tungnath in Garhwal Himalaya. Journal of Biosciences 25, 367-378.

Negi HR, Gadgil M. 2002 - Cross-taxon surrogacy of biodiversity in the Indian Garhwal Himalaya. Biological Conservation 105, 143-155.

Negi HR, Upreti DK. 2000 - Species diversity and relative abundance of lichens in Rumbak catchment of Hemis National Park in Ladakh. Current Science 78, 1105-1112.

Orange A, James PW, White FJ. 2001 - Microchemical methods for the identification of lichens. British Lichen Society, London.

Pinokiyo A, Singh KP, Singh JS. 2008 - Diversity and distribution of lichens in relation to altitude within a protected biodiversity hot spot, north-east India. Lichenologist 40, 47-62.

Pirintsos SA, Diamantopoulos J, Stamou GP. 1995 - Analysis of the distribution of epiphytic lichens within homogeneous Fagus sylvatica stands along an altitudinal gradient (Mount Olympos, Greece). Vegetatio 116, 33-40.

Rai H. 2012 - Studies on diversity of terricolous lichens of Garhwal Himalaya with special reference to their role in soil stability. PhD Thesis. H.N.B Garhwal University. Srinagar (Garhwal), Uttarakhand, India.

Rai H, Khare R, Gupta RK, Upreti DK. 2012a - Terricolous lichens as indicator of anthropogenic disturbances in a high altitude grassland in Garhwal (Western Himalaya), India. Botanica Orientalis 8, 16-23.

Rai H, Khare R, Nayaka S, Upreti DK. 2011- Lichen synusiae in East Antarctica (Schirmacher Oasis and Larsemann Hills): substratum and morphological preferences. Czech Polar Report $1,65-77$.

Rai H, Khare R, Nayaka S, Upreti DK. 2013 - The influence of water variables on the distribution of Terrricolous lichens in Garhwal Himalayas. In: Kumar P, Singh P, Srivastava RJ (Eds) Souvenir, Water \& Biodiversity, 22 May 2013, International day for biological diversity, Uttar Pradesh State Biodiversity Board 7, 75-83.

Rai H, Khare R, Upreti DK, Ahti T. 2014 - Terricolous Lichens of India: Taxonomic Keys and Description. In: Rai H, Upreti DK (Eds.), Terricolous Lichens in India, Springer New York pp. 17-294.

Rai H, Nag P, Upreti DK, Gupta RK. 2010 - Climate warming studies in alpine habitats of Indian Himalaya, using lichen based passive temperature-enhancing system. Nature and Science 8, $104-106$.

Rai H, Upreti DK, Gupta, RK. 2012b - Diversity and distribution of terricolous lichens as indicator of habitat heterogeneity and grazing induced trampling in a temperate-alpine shrub and meadow. Biodiversity and Conservation 21, 97-113.

Rosentreter R, Rai H, Upreti DK. 2014 - Distribution Ecology of Soil Crust Lichens in India: A Comparative Assessment with Global Patterns. In: Rai H, Upreti DK (Eds.), Terricolous Lichens in India, Springer New York, pp. 21-31.

Saag L, Saag A, Randlane T. 2009 - World survey of the genus Lepraria (Stereocaulaceae, lichenized Ascomycota). Lichenologist 41, 25-60.

Scutari NC, Bertiller MB, Carrera AL. 2004 - Soil-associated lichens in rangelands of northeastern Patagonia. Lichen groups and species with potential as bioindicators of grazing disturbance. Lichenologist 36, 405-412.

Seaward M RD. 1988 - Contribution of lichens to ecosystems. In: Galun M (ed), CRC Handbook of Lichenology, Vol. 2, Boca Raton, CRC Press, pp. 107-129.

Sheard JW. 1968 - Vegetation pattern on a moss-lichen heath associated with primary topographic features on Jan Mayen. Bryologist 71, 21-29.

Shrestha G, St. Clair LL. 2011 - A comparison of the lichen floras of four locations in the Intermountain Western United States. North American Fungi 6, 1-20.

ter Braak CJF, Prentice IC. 1988 - A theory of gradient analysis. Advances in Ecological Research 
$18,271-313$.

Upreti DK. 1998 - Diversity of lichens in India. In: Agarwal SK, Kaushik JP, Kaul KK, Jain AK (Eds.) Perspectives in Environment, APH Publishing Corporation, New Delhi, India, pp.7179.

Upreti DK, Negi HR. 1998 - Lichen flora of Chopta-Tungnath, Garhwal Himalayas, Journal of Economic and Taxonomic Botany 22, 273-286.

Wolf JH. 1993 - Diversity patterns and biomass of epiphytic bryophytes and lichens along an altitudinal gradient in the northern Andes. Annals of the Missouri Botanical Garden 80, 928960.

Zedda L, Kong S-M, Rambold G. 2011 - Morphological groups as a surrogate for soil lichen biodiversity in Southern Africa. In: Bates S.T., Bungartz F, Lücking R, Herrera-Campos MA, Zambrano A (Eds.), Biomonitoring, Ecology, and Systematics of Lichens Festschrift Thomas H. Nash III, Bibliotheca Lichenologica 106, 391-408. J. Cramer in der Gebr. Borntraeger Verlagsbuchhandlung, Stuttgart. 